\title{
Psychometric Evidence of a Multidimensional Measure of Prosocial Behaviors for Spanish Adolescents
}

\section{Maria V. Mestre, Gustavo Carlo, Paula Samper, Ana M. Tur-Porcar \& Ana Llorca Mestre}

To cite this article: Maria V. Mestre, Gustavo Carlo, Paula Samper, Ana M. Tur-Porcar \& Ana Llorca Mestre (2015) Psychometric Evidence of a Multidimensional Measure of Prosocial Behaviors for Spanish Adolescents, The Journal of Genetic Psychology, 176:4, 260-271, DOI: 10.1080/00221325.2015.1052726

To link to this article: http://dx.doi.org/10.1080/00221325.2015.1052726

Accepted author version posted online: 01 Jul 2015.

Submit your article to this journal $₫$

Џ Article views: 63

Q View related articles ¿

View Crossmark data $\asymp$ 


\title{
Psychometric Evidence of a Multidimensional Measure of Prosocial Behaviors for Spanish Adolescents
}

\author{
Maria V. Mestre \\ University of Valencia, Spain \\ Gustavo Carlo \\ University of Missouri \\ Paula Samper, Ana M. Tur-Porcar, and Ana Llorca Mestre \\ University of Valencia, Spain
}

\begin{abstract}
Prior theories and scholars rarely distinguished between distinct forms of prosocial behaviors (i.e., actions intended to benefit others) and most scholars operationalize prosocial behaviors as a global construct. Furthermore, stringent tests of psychometric properties of prosocial behavior measures are rare, especially in countries other than the United States. The present study was designed to examine the structure and functions of a multidimensional measure of prosocial behaviors in youth from Spain. Six hundred and 66 adolescents ( $46 \%$ girls; $M$ age $=15.33$ years, $S D=0.47$ years) from Valencia, Spain, completed the Prosocial Tendencies Measure-Revised (PTM-R), measures of sympathy, perspective taking, and a global measure of prosocial behavior. Results showed that the hypothesized 6-factor structure of the PTM-R had good fit, the best fit model as compared to alternative models, and that there were several unique patterns of relations between specific forms of prosocial behaviors and sympathy, perspective taking, and the global measure of prosocial behavior. Furthermore, although there were significant gender differences in some forms of prosocial behaviors, the structure of the PTM-R was equivalent across gender. Discussion focuses on the implications for theories and measurement of prosocial behaviors.
\end{abstract}

Keywords adolescents, culture, measurement, prosocial behaviors, theory

Many adolescents engage in prosocial behaviors (i.e., actions intended to benefit others) such as volunteerism, sharing, donating, comforting others, and helping. Such behaviors occur across different contexts and for different reasons, and these behaviors have been linked to healthy social functioning and well being (Carlo, 2014; Hart, 2005). Despite the accumulated knowledge regarding prosocial development, recent research indicates the need to examine distinct forms of prosocial behaviors (Padilla-Walker \& Carlo, 2014). Indeed, investigators have shown

Received March 3, 2015; accepted May 15, 2015.

Address correspondence to Gustavo Carlo, Department of Human Development and Family Science, 410 Gentry Hall, University of Missouri, Columbia, MO 65211, USA; carlog@ missouri.edu (e-mail). 
individual and group differences in specific forms of prosocial behaviors, and specific types of prosocial behaviors are linked to specific correlates. This growing body of evidence has important implications for theories and measurement of prosocial development.

Despite the interest in specific forms of prosocial behaviors, progress in this area is relatively slow due to the lack of measures that demonstrate strong psychometric properties (Padilla-Walker $\&$ Carlo, 2014). Most previous scholars studying prosocial behaviors have conceptualized such actions as a relatively global, homogenous construct such that all forms of prosocial behaviors were generally considered equal. There were some exceptions in that some investigators would focus on altruistic actions (i.e., behaviors primarily intended to benefit others without expectation for self reward, often at a high cost to an individual's self) and other researchers focused on examining prosocial behaviors in emergency bystander situations (see e.g., Latane \& Darley, 1970; Staub, 1978). However, many other studies focused on one behavior (e.g., helping someone pick up paper clips) or used questionnaire measures with several items depicting different types of prosocial behaviors (e.g., Rushton, Chrisjohn, \& Fekken, 1981; for reviews, see Carlo, 2014). In these latter studies, researchers would often apply a broad theory of prosocial behavior to guide and interpret their findings. Although one might expect some forms of prosocial behaviors to be positively interrelated, other forms of prosocial behaviors may be nonsignificantly related or negatively related. Moreover, extensive evidence on psychometric properties of most previously developed measures is sorely lacking.

Based on a review of the extant literature, extensive piloting, and focus group data, Carlo et al. developed and validated a multidimensional measure of prosocial behaviors to use with adolescents and young adults from the United States (Carlo, Hausmann, Christiansen, \& Randall, 2003; Carlo, Knight, McGinley, Zamboanga, \& Jarvis, 2010). These scholars identified at least six distinct forms of such behaviors that differ across contexts and motives. Compliant prosocial behaviors refer to actions that assist another person when requested by that person (e.g., when a parent or teacher asks a youth for help). Anonymous prosocial behaviors may occur without others knowing who helped (e.g., donating money inconspicuously). When individuals assist others in emergency or emotionally evocative situations, these are referred to as dire and emotional prosocial behaviors, respectively. Public prosocial behaviors can be observed when individuals help in front of an audience. However, altruistic prosocial behaviors are defined as helping others with little or no expectation for self rewards. The resulting instruments, the Prosocial Tendencies Measure (PTM; Carlo \& Randall, 2002) and Prosocial Tendencies Measure-Revised (PTM-R; Carlo et al., 2003), are not designed to characterize all possible types of prosocial behaviors but rather an individual's disposition to engage in six relatively common types of prosocial behaviors.

There is increased use of the PTM-R with adolescents in research and various psychometric properties of the PTM-R have been investigated. For example, there is accumulated supportive evidence of the six-factor structure of the PTM-R, internal consistencies of each scale, and testretest reliabilities (Carlo et al., 2003; Carlo et al., 2010). Furthermore, several different forms of validity have been documented, including discriminant, construct, and convergent validities (Carlo et al., 2003; Eisenberg et al., 2009; Hardy, Carlo, \& Roesch, 2010) across samples of European Americans, U.S. Mexican adolescents (Armenta, Knight, Carlo, \& Jacobson, 2011; Calderón, Knight, \& Carlo, 2011; Carlo et al., 2010) and in youth from Taiwan (Shen, Carlo, \& Knight, 2013), Argentina (McGinley, Opal, Richaud, \& Mesurado, 2014), and Indonesia (Eisenberg et al., 2009). However, further validation of the PTM-R is desirable to facilitate comparative research in different countries. Importantly, comparative research based on measures 
with demonstrated adequate psychometric properties allows for more rigorous tests of the generalizability of multidimensional models of prosocial behaviors across cultures. The present study was designed to examine the psychometric properties of the PTM-R to use with a sample of adolescents from Spain.

Spain is a modern social democracy located in Western Europe and a member of the European Union. Generally characterized as a society that considers the family and religion (predominantly Christianity) as major and central social institutions (Centro de Investigaciones Sociológicas, 2004; Samper, 1999), Spain ranks somewhat higher on individualism, and somewhat lower in masculinity, than other Latino countries, such as Venezuela, Mexico, Colombia, Argentina, and Brazil (Hofstede, 2001; Oyserman, Coon, \& Kemmelmeier, 2002). Moreover, according to the Globe study (House et al., 2004), Spain clusters into the Latin-European group along with Israel, Italy, France, Portugal, and (French speaking) Switzerland. Among these countries, Spain ranks relatively high on performance and team orientation, and an autonomous leader style (i.e., independent, individualistic, and self-centric approach to leadership). On the other hand, pride, loyalty, collaboration, team cohesiveness, and common purpose or goals are highly valued (Chhokar, Brodbeck, \& House, 2007; House et al., 2004). Thus, Spain is substantially lower on individualism than the United States and is moderately high on individualism as compared to other industrialized countries (Hofstede, 2001; Oyserman et al., 2002). Finally, although Spain is undoubtedly experiencing changes as a result of population growth and economic changes, some scholars deem Spain a moderately collectivist, feminine-oriented society (de Prada, Actis, \& Pereda, 2005; Fernández-Berrocal, Salovey, Vera, Ramos, \& Extremera, 2001). The general collectivist orientation and central roles of family and religion in Spain are characteristics associated with prosocial values and behaviors in other Latino cultures (Carlo $\&$ de Guzman, 2009); thus, in and of itself, Spain provides an interesting context for studying prosocial behaviors. Moreover, assumptions concerning the adequacy of the PTM-R is concerning given the unique cultural orientation of Spain as compared to the United States and other countries.

Although one might expect culture group differences in specific forms of prosocial behaviors, there is no strong conceptual reason to expect that the structure and psychometric properties of the PTM-R should differ among Spanish adolescents (e.g., as compared to adolescents from the United States). Thus, based on theory and prior research (on youth from other countries), we hypothesized that a six-factor structure of the PTM-R should have adequate fit. Furthermore, the six-model structure was expected to demonstrate the best fit as compared to alternative models (such as a one-factor model). However, based on gender socialization theories and prior findings, gender differences in prosocial behaviors were expected, such that girls would report more emotional, dire, altruistic, compliant, and anonymous prosocial actions whereas boys would report more public prosocial actions (see Carlo, 2014). Despite the expected gender differences, however, we hypothesized that the proposed six-factor structure would fit equally well across boys and girls.

Developmental scholars have identified perspective taking and sympathy as important correlates of prosocial behaviors (Eisenberg, Fabes, \& Spinrad, 2006; Hoffman, 2000). Perspective taking is defined as understanding the thoughts, feelings, and social situations of others. Generally, perspective taking is deemed to facilitate prosocial behaviors (though sometimes perspective taking may result in taking advantage of, or harming, another). Similarly, sympathy (or empathic concern), which refers to feelings of concern or sorrow for a distressed other, is linked positively 
to prosocial behaviors. Previous research demonstrates relatively consistent positive relations between both perspective taking and sympathy and prosocial behaviors (see Carlo, 2014; Eisenberg et al., 2006). However, we expected a differential pattern of relations between perspective taking and sympathy and prosocial behaviors. For example, given that perspective taking may be less necessary to discern need in emergency, emotionally evocative, or compliant situations, perspective taking was expected to be relatively weakly related to these prosocial behaviors. In contrast, sympathy was expected to be particularly strongly related to helping in dire, emotional, and altruistic situations, and negatively (or unrelated) related to public helping. These expected differential pattern of relations between specific forms of prosocial behaviors and specific gender, perspective taking, and sympathy provide tests of construct validity. To test convergent validity, we also examined the relations between a commonly used global measure of prosocial behaviors and the PTM-R. Thus, the study provides the first and strongest examination of the notion of the multidimensionality of prosocial behaviors and the various psychometric properties of the PTM-R in a sample of adolescents from Spain.

\section{METHOD}

\section{Participants and Procedure}

The sample was composed of 666 students ( 297 girls, $M$ age $=15.32$ years, $S D=0.47$ years) from public and private schools located in different geographic zones within the school district of Valencia, Spain. Most were from two-parent households (73\%; 27\% single-parent households). Mothers' education level was 35\% less than high school diploma (25\% for fathers), $37 \%$ high school diploma or equivalent ( $28 \%$ for fathers), and $26 \%$ at least some university education (34\% for fathers). The majority self-identified from Spain (76\%) and others were from Latin America (i.e., 7\% from Ecuador, 2\% Colombia, 2\% Bolivia) and from Eastern European countries (e.g., $2 \%$ Romania). Signed informed consent from the parents was required to participate. The signed consent forms were returned in a sealed envelope to the schools and collected by the experimenters. Trained experimenters administered the surveys in classrooms (completion time of approximately $50 \mathrm{~min}$ ) and all participants were provided an educational debriefing.

\section{Measures}

The measures of perspective taking, sympathy, and the global prosocial behavior have been translated, adapted for use, and validated in samples of adolescents from Spain (e.g., Del Barrio, Moreno, \& Lopez, 2001; Mestre, Samper, \& Frías, 2002;Mestre, Samper, Frias, \& Tur, 2009).

\section{Prosocial Behaviors}

The PTM-R (Carlo et al., 2003) was designed to assess six different prosocial behaviors: emotional, altruism, dire, compliant, public and anonymous. The measure was translated and back translated independently by bilingual experts on prosocial behaviors (the primary coauthors). 
There were few minor discrepancies, which were discussed and resolved by the research team. The measure was administered to adolescents and includes 21 Likert-type items with five response options ranging from 1 (does not describe you at all) to 5 (describes you very well). Public prosocial behaviors were defined as behaviors intended to benefit others enacted in the presence of others (three items; sample item: "I can help others best when people are watching me"). Anonymous prosocial behavior is the tendency to help others without other people's knowledge (four items; "I think that helping others without them knowing is the best type of situation"). Dire prosocial behavior is helping others under emergency or crisis situations (three items; "I tend to help people who are in real crisis or need"). Emotional prosocial behaviors are enacted under emotionally evocative situations (five items; "It makes me feel good when I can comfort someone who is very upset"). Compliant prosocial behavior is helping others when asked to (two items; "When people ask me to help them, I don't hesitate"). Altruistic refers to helping others with little or no perceived direct, explicit reward to the self (four items; item is reverse scored: "I believe that donating goods or money works best when I get some benefit"). Cronbach's alphas in the present sample ranged from .61 to .79 .

\section{Global Measure of Prosocial Behavior}

The global measure of prosocial behavior (Caprara \& Pastorelli, 1993; Del Barrio, Moreno, \& Lopez, 2001) contains 10 items, summed and averaged, (Cronbach's alpha $=.85$, present sample) that assess helping behaviors (e.g., "I am caring toward my friends.") on a 3-point Likert-type scale, anchored by 1 (never) to 3 (frequently).

\section{Sympathy and Perspective Taking}

The affective and cognitive components of empathy were assessed using the sympathy (i.e., empathic concern) and perspective taking subscales of the Interpersonal Reactivity Index (Davis, 1983). Each scale has items such as "The problems of the others worry me" (sympathy; $\alpha=.53$; seven items, present sample) and "When I must decide, I listen to different opinions" (perspective taking; $\alpha=.64$; present sample; five items after dropping two reversed scored items because of low item loading) on a 5-point Likert-type scale ranging from 1 (does not describe you well) to 5 (describes you very well).

\section{RESULTS}

\section{Main Data Analytic Approach}

The main analyses were conducted using the Analysis of Moments Structure (AMOS) statistical software (Arbuckle, 2007). First, we tested the proposed six-factor model in the overall sample using confirmatory factor analysis. Second, we tested several alternative factor structures. And third, we directly compared the model fit of the proposed six-factor structure model with each of the alternative models. In all model examinations, fit is considered good if the comparative 
TABLE 1

Descriptives and Correlations Among the Main Study Variables

\begin{tabular}{|c|c|c|c|c|c|c|c|c|c|c|}
\hline & 1 & 2 & 3 & 4 & 5 & 6 & 7 & 8 & 9 & 10 \\
\hline \multicolumn{11}{|l|}{ 1. Gender } \\
\hline 2. Public & $-.20^{* * *}$ & & & & & & & & & \\
\hline 3. Emotional & $.23^{* * *}$ & $.10^{* *}$ & & & & & & & & \\
\hline 4. Altruistic & $.22^{* * *}$ & $-.51^{* * *}$ & $-.08^{*}$ & & & & & & & \\
\hline 5. Dire & $.09^{*}$ & $.12^{* *}$ & $.65^{* * *}$ & -.05 & & & & & & \\
\hline 6. Anonymous & -.08 & $.21^{* * *}$ & $.21^{* * *}$ & $-.17^{* * *}$ & $.25^{* * *}$ & & & & & \\
\hline 7. Compliant & $.21^{* * *}$ & -.03 & $.61^{* * *}$ & $.10^{* *}$ & $.54^{* * *}$ & $.18^{* * *}$ & & & & \\
\hline 8. Perspective taking & $.14^{* *}$ & $.10^{*}$ & $.40^{* * *}$ & -.01 & $.35^{* * *}$ & $.15^{* *}$ & $.37^{* * *}$ & & & \\
\hline 9. Sympathy & $.35^{* * *}$ & -.02 & $.47^{* * *}$ & $.17^{* * *}$ & $.40^{* * *}$ & $.10^{*}$ & $.34^{* * *}$ & $.40^{* * *}$ & & \\
\hline 10. Global prosocial behavior & $.26^{* * *}$ & $-.08^{*}$ & $.36^{* * *}$ & $.12^{* *}$ & $.21^{* * *}$ & .01 & $.36^{* * *}$ & $.28^{* * *}$ & $.33^{* * *}$ & \\
\hline$M$ & 1.46 & 2.04 & 3.46 & 3.54 & 3.42 & 2.40 & 3.72 & 3.23 & 3.48 & 2.29 \\
\hline$S D$ & 0.50 & 0.92 & 0.84 & 0.88 & 0.85 & 0.84 & 0.98 & 0.61 & 0.57 & 0.27 \\
\hline
\end{tabular}

Note. Public, emotional, altruistic, dire, anonymous, and compliant refer to prosocial behavior scales from the Prosocial Tendencies Measure-Revised (PTM-R). Girls were scored as 2, boys were scored as 1. All tests were two-tailed.

${ }^{*} p<.05 .^{* *} p<.01 .^{* * *} p<.001$.

fit index (CFI) is $>.95$ (or .90 for adequate fit), the root mean square error of approximation (RMSEA) is $<.06$ (.08 for adequate fit), and the standardized root mean square residual is $<.10$ (Hu \& Bentler, 1999; Kline, 1998). Because the above model comparisons do not represent true nested models, the Akaike information criterion (AIC), Bayesian information criterion (BIC), and expected cross-validation index (ECVI) were used to assess relative fit. In general, the AIC, BIC, and ECVI correct model fit for model complexity, and lower values indicate better model fit (Brown, 2006).

Two six-factor models were first tested. The first (proposed) six-factor model assumed that all six subscales were interrelated, the second six-factor model assumed that only some subscales were interrelated (based on initial correlations among the subscales, see Table 2). Then, we created three additional alternative models to use as direct comparisons to the proposed six-factor model. The first alternative model was based on traditional approaches to studying prosocial behaviors and resulted in a one-factor (global) model. A second alternative model was based on the notion that highly correlated factors can indicate construct overlap (Brown, 2006). For this

TABLE 2

Tests of the Proposed and Alternative Latent Factor Models

\begin{tabular}{lcccccc}
\hline & $\chi^{2}(\mathrm{df})$ & CFI & RMSEA & AIC & BIC & ECVI \\
\hline Six-factor model (all correlated) & $501.13(174)$ & .91 & .05 & 657.13 & 662.68 & 1.03 \\
Six-factor model (some correlated) & $564.68(182)$ & .90 & .06 & 704.68 & 709.66 & 1.10 \\
Three-factor model & $627.44(186)$ & .88 & .06 & 759.44 & 764.13 & 1.19 \\
Two-factor model & $959.30(188)$ & .79 & .08 & 1087.30 & 1091.85 & 1.70 \\
One-factor model & $1910.20(189)$ & .53 & .12 & 2036.20 & 2040.68 & 3.18 \\
\hline
\end{tabular}

Note . CFI = comparative fit index; RMSEA = root meant square error of approximation; AIC = Akaike information criterion; $\mathrm{BIC}=$ Bayesian information criterion; $\mathrm{ECVI}=$ expected cross-validation index. 
model, we created a three-factor model that combined emotional, dire, and compliant into one factor, combined public and altruistic into a second factor, and left anonymous as a third factor. Finally, based on the conceptual distinction between prosocial tendencies expressed in different contexts (emotional, dire, compliant, anonymous into one factor) versus those expressed for different motives (public and altruistic into a distinct factor), a two-factor alternative structure was also tested.

Upon establishing adequate fit of the proposed six-factor model, measurement equivalence analyses were conducted for gender. The present study focused on item, functional, and scalar equivalence (Hui \& Triandis, 1985; Knight, Tein, Prost, \& Gonzales, 2002). Item equivalence was assessed by exploring the factorial invariance of the PTM-R, while functional and scalar equivalence was assessed by exploring the construct validity equivalence of the PTM-R. In addition to the practical fit indices (CFI, RMSEA), the chi-square difference test $\left(\Delta \chi^{2}\right)$ was also used to evaluate the relative model fit. However, as chi-square criteria are sensitive to trivial modifications of fit, other criteria were implemented (see Browne \& Cudeck, 1993; Cheung \& Rensvold, 2002). Thus, if the chi-square difference was accompanied by (a) a considerable drop in the practical fit indices and/or (b) a large (i.e., > 3) ratio of chi-square difference to degrees of freedom $\left(\Delta \chi^{2}: \Delta d f\right)$, modification indices were used to determine which parameters were significantly different across the two groups. Once these parameters were allowed to be freely estimated across the groups, partial invariance/equivalence could be attained if model fit indices signified adequate fit.

\section{Tests of the Proposed Six-Factor Structure}

For the overall sample, the fully correlated, proposed 21-item six-factor model fit good according to fit indices, $\chi^{2}(174, N=666)=501.13, p<.001, \mathrm{CFI}=.91$, RMSEA $=.05$. Factor loadings for this model were all positive, significant, and averaged .63 (ranging from .48 to .77 , with one exception of .30). A six-factor model, which allowed for some subscales to be uncorrelated (based on the interrelations shown in Table 1), had adequate fit according to fit indices, $\chi^{2}(182$, $N=666)=564.68, p<.001, \mathrm{CFI}=.90$, RMSEA $=.06$. Also, based on relative fit indices, a chi-square difference test showed that the fully correlated six-factor model had significantly better fit than the partially correlated six-factor model, $\Delta \chi^{2}(8, N=666)=63.55, p<.001$ (AIC $=657.13$ vs. 704.68 , respectively; $\mathrm{BIC}=662.58$ vs. 709.66 , respectively; $\mathrm{ECIV}=1.03$ vs. 1.10 , respectively).

Results of chi-square tests further showed that the proposed six-factor model was a significantly $(p<.001)$ better fit than one-, $\Delta \chi^{2}(15, N=666)=1409.07$; two-,$\Delta \chi^{2}(14, N=666)=458.17$; and three-factor, $\Delta \chi^{2}(12, N=666)=126.31$, alternative models. The AIC, BIC, and ECVI values were all lower for the proposed six-factor model than all other models (see Table 2). Therefore, as hypothesized, the fully correlated, six-factor structure model was retained as the best fitting structure model.

\section{Gender Measurement Equivalence Test}

To examine whether the proposed six factor model was equivalent across boys and girls, a multiple group analysis was conducted. For these analyses, the chi-square coefficient for a constrained model was tested against the chi-square coefficient of an unconstrained model. The constrained 
model, $\chi^{2}(363, N=666)=676.487$, and unconstrained model, $\chi^{2}(348, N=666)=665.269$, were not significantly different, $\Delta \chi^{2}(15, N=666)=11.218$. Therefore, full factorial invariance for the proposed six factor model was achieved and, as hypothesized, the PTM-R was measurably equivalent across boys and girls.

\section{Descriptives and Construct Validity Analyses}

Table 1 presents the means, standard deviations, and interrelations among the main study variables. Consistent with hypotheses, girls scored higher than boys on emotional, altruistic, dire, and compliant prosocial tendencies, on the global measure of prosocial behavior, and on perspective taking and sympathy. Furthermore, boys scored higher than girls only on public prosocial tendencies.

There were several significant relations between the PTM-R subscales and empathic tendencies and the global measure of prosocial behavior. Specifically, generally as hypothesized, sympathy was positively related to compliant, altruistic, emotional, compliant, anonymous, and dire (but not public) prosocial behaviors. Perspective taking was positively related to all the prosocial behaviors (except altruistic prosocial behaviors) and the magnitude of effect were somewhat unexpectedly similarly in size to that of sympathy. Finally, as expected, the global measure of prosocial behavior was positively related to compliant, altruistic, emotional, and dire prosocial behaviors. Furthermore, the global measure was negatively related to public prosocial behaviors, and not significantly related to anonymous prosocial behaviors.

\section{DISCUSSION}

There was overall support for the internal structure and validity of a multidimensional measure of prosocial tendencies to use with adolescents from Spain. The proposed six-factor model demonstrated good fit, and the best fit as compared to several conceptually and empirically based alternative models. Furthermore, the proposed model showed equivalence across gender. Moreover, supportive validity evidence regarding expected relations between PTM-R subscales and gender, sympathy, and a global measure of prosocial behavior was presented. These findings demonstrate the most stringent evidence of the factor structure of the PTM-R to use with youth in Spain. These findings have important implications for the notion of multidimensional models of prosocial behaviors.

The present findings demonstrate the first evidence on the factor structure of the PTM-R in a sample of youth from Spain and the proposed six-factor structure held across boys and girls. As expected, confirmatory factor analysis of the PTM-R suggests that the measure assesses six distinct though modestly correlated forms of prosocial behaviors: compliant, dire, emotional, anonymous, public, and altruistic. These findings add to the sparse but growing evidence that the proposed six factor structure of the PTM-R is the best fit model as compared to several alternative models, including a global, one factor model (Carlo et al., 2010; McGinley et al., 2014). These findings are also in accord with previous research in other ethnic samples (Carlo et al., 2010; McGinley et al., 2014), which suggests that the proposed six-factor structure is generalizable to youth from a relatively collectivistic, family, and (Christian) religious-oriented society. 
Conceptually, the multidimensionality of prosocial behaviors suggests that there may be unique antecedents, correlates, developmental trajectories, and consequences of specific forms of prosocial behaviors. The fact that we found a distinct pattern of relations between sympathy, perspective taking, gender, and the six PTM-R subscales is consistent with the previous assertion, and yields supportive evidence regarding the construct and convergent validity of the PTM$\mathrm{R}$ in Spanish youth. Correlational analyses demonstrated positive significant relations between both sympathy and perspective taking and emotional, dire, and compliant prosocial tendencies. However, there were no significant relations between sympathy and public prosocial behaviors. Because public prosocial behaviors have been linked to approval-oriented moral reasoning, scholars have suggested and found evidence that such helping may be motivated by the desire to gain the approval of others (Carlo \& Randall, 2002). This self-oriented motive would be expected to be less likely to be associated with a concern for others' plight. Moreover, although altruistic helping was positively associated with sympathy, it was not significantly related to perspective taking. Previous researchers have suggested that perspective taking can sometimes lead to actions that are done to take advantage of others and that may even be harmful (Carlo, 2014). In contrast, because sympathy is considered an intrinsic concern for others, it is relevant to understanding altruistically motivated forms of helping (Eisenberg et al., 2006).

Based on Cohen's (1992) guidelines for interpreting magnitude of effect sizes, the global prosocial behavior measure correlated with the specific measures as follows: (a) a moderate positive correlation for emotional and compliant, (b) a small to moderate positive correlation for dire, (c) a small positive correlation for altruistic, (d) a small negative correlation for public, and (e) a nonsignificant correlation for anonymous. These findings suggest that the global measure of prosocial behavior primarily assesses emotional and compliant forms of prosocial behaviors, with a relatively lesser assessment of altruistic, dire, and public forms, and no overlap with anonymous helping. A similar pattern of specific relations between a global measure of prosocial behavior and the PTM-R has also been reported in a study of European American youth using a different global measure (e.g., Carlo et al., 2003). It should also be noted that the negative relation between the public prosocial behavior subscale and other types of prosocial behaviors has been consistently found in prior research with Mexican American and European American youth. This latter finding is in accord with the previously noted notion that engaging in public forms of helping may be motivated by a desire to gain others' approval and self-presentational demands (Carlo, 2014).

Several gender differences in prosocial behaviors provided additional discriminant validity evidence. Consistent with prior research (Carlo, 2014; Eagly \& Crowley, 1986), girls scored higher than boys on emotional, dire, altruistic, and compliant forms of prosocial behaviors; whereas boys scored higher than girls on public prosocial behaviors. Boys have been reported to engage in public forms of helping more so than girls, which has been linked to gaining social approval and may enhance their social status in groups. The forms of helping that girls have been linked to are consistent with feminine-typed conduct and actions associated with sympathy responding, which is often encouraged by societal agents. Boys, on the other hand, may be discouraged or scorned if they express sympathy or feminine-typed forms of conduct. Because the PTM-R demonstrates measurement equivalence across Spanish boys and girls, the present pattern of gender differences are unlikely due to measurement artifact.

Despite the generally expected pattern of findings, some caution is needed in interpreting the results. First, the sample may not be fully representative of adolescents across Spain. Because 
there are differences both within and between cultures, it would be desirable to replicate the results and evaluate the PTM-R in samples from other regions of the country. Second, the PTM-R may be subject to self-presentational demands. Although previous research suggests that the PTM-R correlates weakly or nonsignificantly with measures of social desirability (Carlo et al., 2003), future studies should assess prosocial behaviors using multiple reporters or methods (e.g., observations). Third, a reasonable next step is to further examine the generalizability of the proposed six-factor structure of the PTM-R in cross-cultural research that directly compares the PTM-R in youth from various countries. Finally, further validation efforts are needed to replicate the present findings. For example, given the relatively low or marginally adequate reliabilities of some of the measures, future validation studies using different measures of empathic tendencies (e.g., facial expressions, physiological measures) and prosocial behaviors (e.g., observations, multiple reporters) would be desirable. Despite these limitations, the present findings suggest that the PTM-R is useful as a multidimensional measure of prosocial behavior in Spanish youth.

\section{FUNDING}

Funding support was provided by an I + D Projects for Research Teams of Excellence, PROMETEO Program (Reference: PROMETEO/2011/2009), Department of Education, Generalitat Valenciana and I + D Project funded by the Ministry of Science and Innovation (reference PSI2011-27158) to Maria V. Mestre. Manuscript writing was supported by an Invited Visiting Scholar Fellowship to Gustavo Carlo from the University of Valencia.

\section{AUTHOR NOTES}

Maria V. Mestre is Professor of Psychology (Psychological Processes) in the Department of Basic Psychology at the University of Valencia (Spain). Her main research interest are in cognitive and emotional processes, social competence on prosocial development and the role of parenting styles. She has several ongoing projects focusing on protective factors and vulnerability in adolescence. Gustavo Carlo is the Millsap Professor of Diversity and Multicultural Studies in the Department of Human Development and Family Science at the University of Missouri. His main research interests are in the roles of culture, personality, and parenting on prosocial development. He has several ongoing projects focusing on Latinos in various regions of the U.S., Spain, and Argentina. Paula Samper is Professor in the Department of Basic Psychology at the University of Valencia in Spain. Her research interests are in basic psychological processes and prosocial behaviors under the directorship of Dr. Maria Vicenta Mestre Escriv. Specifically, Dr. Samper's research focuses on family correlates (such as family cohesion and conflict) of prosocial and aggressive behaviors in youth from an multicultural context. Ana M. Tur-Porcar is currently an Associate Professor at the University of Valencia in Spain. She holds a PhD from University of Valencia. Her research focuses on understanding parenting, prosocial and aggressive behaviors. She has published papers in journals such as: Personality and Individual Differences, Journal of Cross-cultural Psychology, Journal of Adolescence, and the European Journal of Developmental Psychology. Ana Llorca Mestre is Assistant Professor in the Department of Personality, Psychological Assessment and Treatment at the University of Valencia. Her main research interest are in adolescents' aggressive 
and delinquent behaviors, intra-family violence and violence from adolescents to parents, and the role of empathy and prosocial development.

\section{REFERENCES}

Arbuckle, J. L. (2007). Amos 16.0 user's guide. Chicago, IL: SPSS, Amos Development Corporation.

Armenta, B. E., Knight, G. P., Carlo, G., \& Jacobson, R. (2011). The relation between ethnic group attachment and prosocial tendencies: The mediating role of ethnic specific cultural values. European Journal of Social Psychology, $41,107-115$.

Brown, T. A. (2006). Confirmatory factor analysis for applied research. New York, NY: Guilford Press.

Browne, M. W., \& Cudeck, R. (1993). Alternative ways of assessing model fit. In K. A. Bollen \& J. S. Long (Eds.), Testing structural equation models (pp. 136-162). Beverly Hills, CA: Sage.

Calderón, C. O., Knight, G. P., \& Carlo, G. (2011). The socialization of prosocial behaviors among Mexican American adolescents: The role of familism. Cultural Diversity and Ethnic Minority Psychology, 17, 98-106.

Caprara, G. V., \& Pastorelli, C. (1993). Early emotional instability, prosocial behavior, and aggression: Some methodological aspects. European Journal of Personality, 7, 19-36.

Carlo, G. (2014). The development and correlates of prosocial moral behaviors. In M. Killen \& J. G. Smetana (Eds.), Handbook of moral development (2nd ed.). New York, NY: Psychology Press.

Carlo, G., \& de Guzman, M. R. T. (2009). Theories and research on prosocial competencies among US Latinos/as. In F. Villaruel, G. Carlo, M. Azmitia, J. Grau, N. Cabrera, \& J. Chahin (Eds.), Handbook of U.S. Latino psychology (pp. 191-211). Thousand Oaks, CA: Sage.

Carlo, G., Hausmann, A., Christiansen, S., \& Randall, B. A. (2003). Sociocognitive and behavioral correlates of a measure of prosocial tendencies for adolescents. Journal of Early Adolescence, 23, 107-134.

Carlo, G., Knight, G. P., McGinley, M., Zamboanga, B. L., \& Jarvis, L. (2010). The multidimensionality of prosocial behaviors: Evidence of measurement invariance in early Mexican American and European American adolescents. Journal of Research on Adolescence, 20, 334-358.

Carlo, G., \& Randall, B. A. (2002). The development of a measure of prosocial behaviors for late adolescents. Journal of Youth and Adolescence, 31, 31-44.

Centro de Investigaciones Sociológicas. (2004). Revista Española de investigaciones sociológicas [Spanish journal of sociological studies]. Madrid, Spain: Ministerio de la Presidencia.

Cheung, G. W., \& Rensvold, R. B. (2002). Evaluating goodness-of-fit indexes for testing measurement invariance. Structural Equation Modeling, 9, 233-255.

Chhokar, J. S., Brodbeck, F. C., \& House, R. J. (2007). Culture and leadership across the world: A GLOBE report of in-depth studies of the cultures of 25 countries. Mahwah, NJ: Erlbaum.

Cohen, J. (1992). A power primer. Psychological Bulletin, 112, 155-159.

Davis, M. H. (1983). Measuring individual differences in empathy: Evidence for a multidimensional approach. Journal of Personality and Social Psychology, 44, 113-126.

De Prada, M. A., Actis, W., \& Pereda, C. (2005). Inmigracion y vivienda en España [Immigration and life in Spain]. Colectivo Ioé. Madrid, Spain: Ministerio de Trabajo y Asuntos Sociales, Subdirección General de Información Administrativa y Publicaciones.

Del Barrio, V., Moreno, C., \& López, R. (2001). Evaluación de la agresión e inestabilidad emocional en niños españoles y su relación con la depression [Evaluation of aggression and emotional instability in Spanish children and its relations to depression]. Clínica y Salud, 13, 33-50.

Eagly, A. H., \& Crowley, M. (1986). Gender and helping behavior: A meta-analytic review of the social psychological literature. Psychological Bulletin, 100, 283-308.

Eisenberg, N., Fabes, R. A., \& Spinrad, T. L. (2006). Prosocial development. In N. Eisenberg, W. Damon, \& R. M. Lerner (Eds.), Handbook of child psychology: Social, emotional, and personality development (Vol. 3, 6th ed., pp. 646-718). Hoboken, NJ: Wiley.

Eisenberg, N., Sallquist, J., French, D. C., Purwono, U., Suryanti, T., \& Pidada, S. (2009). The relations of majorityminority group status and having an other-religion friend to Indonesian youths' socioemotional functioning. Developmental Psychology, 45, 248-259. 
Fernández-Berrocal, P., Salovey, P., Vera, A., Ramos, N., \& Extremera, N. (2001). Cultura, inteligencia emocional percibida y ajuste emocional: Un studio preliminar [Culture, perceived emotional intelligence, and emotional adjustment: A preliminary study]. Revista Electrónica de Motivación y Emoción, 4, 8-9.

Hardy, S., Carlo, G., \& Roesch, S. C. (2010). Links between adolescents' expected parental reactions and prosocial behavioral tendencies: The mediating role of prosocial values. Journal of Youth and Adolescence, 39, 85-94.

Hart, D. (2005). The development of moral identity. In G. Carlo \& C. P. Edwards (Eds.), The 51st annual symposium on motivation: Moral motivation across the life span. Lincoln, NE: University of Nebraska Press.

Hoffman, M. L. (2000). Empathy and moral development: Implications for caring and justice. Cambridge, England: Cambridge University Press.

Hofstede, G. H. (2001). Culture's consequences (2nd ed.). Thousand Oaks, CA: Sage.

House, R. J., Hanges, P. J., Javidan, M., Dorfman, P. W., \& Gupta, V. (2004). Culture, leadership, and organizations: The GLOBE study of 62 societies. Thousand Oaks, CA: Sage.

Hu, L., \& Bentler, P. M. (1999). Cutoff criteria for fit indices in covariance structure analysis: Conventional criteria versus new alternatives. Structural Equation Modeling: An Interdisciplinary Journal, 6, 1-55.

Hui, C. H., \& Triandis, H. C. (1985). Measurement in cross-cultural psychology: A review and comparison of strategies. Journal of Cross-Cultural Psychology, 16, 131-152.

Kline, R. B. (1998). Principles and practice of structural equation modeling. New York, NY: Guilford Press.

Knight, G. P., Tein, J.-Y., Prost, J. H., \& Gonzales, N. A. (2002). Measurement equivalence and research on Latino children and families: The importance of culturally informed theory. In J. Contreras, K. Kears, \& A. Neal-Barnett (Eds.), Latino children and families in the United States: Current research and future directions (pp. 181-201). Westport, CT: Praeger.

Latané, B., \& Darley, J. M. (1970). The unresponsive bystander: Why doesn't he help? New York, NY: Appleton-CenturyCrofts.

McGinley, M., Opal, D., Richaud, M. C., \& Mesurado, B. (2014). Cross-cultural evidence of multidimensional prosocial behaviors: An examination of the prosocial tendencies measure (PTM). In L. M. Padilla-Walker \& G. Carlo (Eds.), Prosocial behavior: A multidimensional approach. New York, NY: Oxford University Press.

Mestre, V., Samper, P., \& Frías, M. D. (2002). Procesos cognitivos y emocionales predictores de la conducta prosocial y agresiva: La empatía como factor modulador [Cognitive processes and emotional predictors of prosocial behavior and aggression: Empathy as an influential factor]. Psicothema, 14, 227-232.

Mestre, V., Samper, P., Frias, M. D., \& Tur, A. (2009). Are women more empathetic than men? A longitudinal study in adolescence. The Spanish Journal of Psychology, 12, 76-83.

Oyserman, D., Coon, H. M., \& Kemmelmeier, M. (2002). Rethinking individualism and collectivism: Evaluation of theoretical assumptions and meta-analyses. Psychological Bulletin, 128, 3-72.

Padilla-Walker, L. M., \& Carlo, G. (2014). Prosocial behavior: Past, present, and future. In L. M. Padilla-Walker \& G. Carlo (Eds.), Prosocial behavior: A multidimensional approach. New York, NY: Oxford University Press.

Rushton, J. P., Chrisjohn, R. D., \& Fekken, G. C. (1981). The altruistic personality and the self-report altruism scale. Personality and Individual Differences, 2, 293-302. http://dx.doi.org/10.1016/0191-8869(81)90084-2.

Samper, P. (1999). Variables familiares y formación en valores [Family variables and the development of values]. Doctoral dissertation, University of Valencia, Spain.

Shen, Y.-L., Carlo, G., \& Knight, G. P. (2013). Relations between parental disciplines, empathy-related traits, and prosocial moral reasoning: A multi-cultural examination. Journal of Early Adolescence, 33, 994-1021.

Staub, E. (1978). Positive social behavior and morality: Social and personal influences (Vol. 1). New York, NY: Academic Press. 